Bond University

Research Repository

\title{
Mixed Reality Multimodal Learning Analytics
}

Cowling, Michael A.; Birt, James R.

Published in:

Encyclopedia of Educational Innovation

DOI:

10.1007/978-981-13-2262-4_149-1

Licence:

Other

Link to output in Bond University research repository.

Recommended citation(APA):

Cowling, M. A., \& Birt, J. R. (2020). Mixed Reality Multimodal Learning Analytics. In M. Peters, \& R. Heraud (Eds.), Encyclopedia of Educational Innovation Springer. https://doi.org/10.1007/978-981-13-2262-4_149-1

\section{General rights}

Copyright and moral rights for the publications made accessible in the public portal are retained by the authors and/or other copyright owners and it is a condition of accessing publications that users recognise and abide by the legal requirements associated with these rights.

For more information, or if you believe that this document breaches copyright, please contact the Bond University research repository coordinator. 


\title{
Chapter Title:
}

Mixed Reality Multimodal Learning Analytics

\section{Authors:}

A/Prof. Michael Cowling

CQUniversity

Brisbane, Queensland, Australia

m.cowling@cqu.edu.au

Corresponding (No)

\author{
A/Prof. James Birt \\ Bond University \\ Gold Coast, Queensland, Australia \\ jbirt@bond.edu.au \\ Corresponding (Yes)
}

\section{Introduction:}

There is growing evidence that mixed reality visualisation methods improve learning outcomes especially in spatial disciplines. But, often these studies are focused on post evaluation of a single user visualisation experience and self-reporting.

With almost all interactions within mixed reality environments never recorded or reflected upon, this leaves vital analytics of the learning process lost to the learning stakeholders. This is especially true when trying to understand how learners navigate, interact and communicate within mixed reality learning environments. Compounding this is the increasing need for synchronous communication between learning stakeholders in the mixed reality environments and growing importance on multimodal data recording.

As education shifts from an educator-learner centric model to a multi-disciplinary stakeholder team, there will be growing importance to enable biometric, spatial and reflective multimodal analytics that can be evaluated not only by humans within the stakeholder team but artificial intelligent agents. This recording of multimodal data will also bring with it important ethical and legal issues around privacy, security and machine learning bias.

This chapter will contribute to the theme of immersive technologies and mixed reality innovation in education by exploring current methods that can enable multimodal learning analytics within mixed reality learning applications but also reflecting on the issues we currently face with respect to data storage, privacy, security and machine learning capability and bias.

\section{Situating Learning Analytics in Mixed Reality:}

The reality-virtuality continuum proposed by Milgram and Kishino (1994) and illustrated in figure 1 allows for the position of different applications along the continuum from physical environments on the left-hand side to virtual reality (VR) on the right-hand side. The centre of the continuum or mixed reality is where the physical world is augmented by the virtual or augmented reality (AR) moving towards the virtual world augmented by the physical reality or augmented virtuality (AV). 


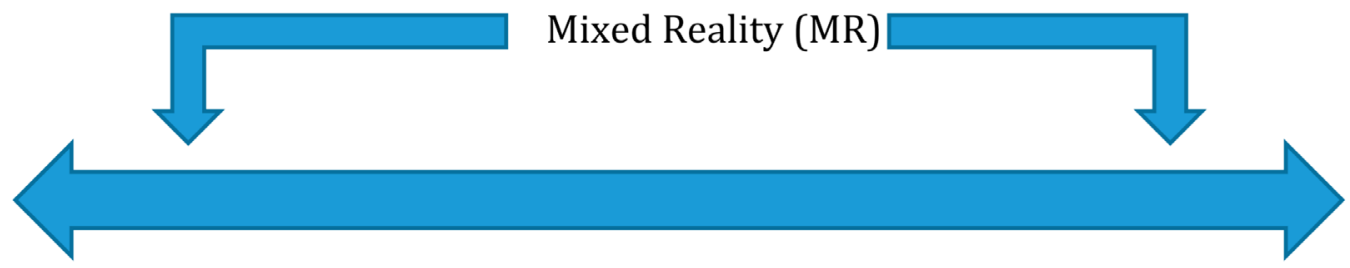

Real

Augmented Reality (AR)

Augmented Virtuality (AV)

Virtual Environment

Figure 1. Reality-virtuality continuum (adapted from Milgram and Kishino (1994)

Mixed Reality Multimodal Learning Analytics (MRMLA) refers to the process of interpreting multimodal spatial data obtained from a MR simulation or application to generate analytics that can be used to evaluate student educational learning outcomes. MR presents an excellent opportunity to facilitate this, as the spatial nature of the simulation or application provides additional data on the student learning process that cannot be obtained from a single mode representation (be that physical or virtual) on the extremes of the continuum.

To leverage MRMLA, a simulation must therefore aim to target the middle of the reality-virtuality continuum (figure 1), providing a perfect mix of the physical and the digital that allows a learner to seamlessly switch between modes. MRMLA could be said to sit firmly in the centre of the continuum, containing digital and physical information, and using objects represented physically and augmented digitally to allow users to manipulate the learning space and assisting the stakeholders in the learning process with valuable data to inform decision making.

\section{True Mixed Reality}

Whilst often positioned as only applying to certain technology in certain situations, true mixed reality is device agnostic and free of technical considerations such as whether the host device is tethered to a computer (tethered mixed reality) or self-contained in a portable device (mobile mixed reality); whether the device is controlled by a joystick or controller or via a new interface mechanic (such as a gaze or teleport mechanic); or whether the mixed reality simulation is presented in a headset (often referred to as immersive mixed reality) or via holding up a device and looking through it (windowed virtual reality). Yet despite this, all of these are important considerations for a mixed reality simulation designer, as the choices they make in these situations can affect how the simulation or application is used (Birt \& Cowling 2018). To illustrate this point figure 2 highlights how the same learning artefact can be represented and engaged with using different devices and visualization modes.

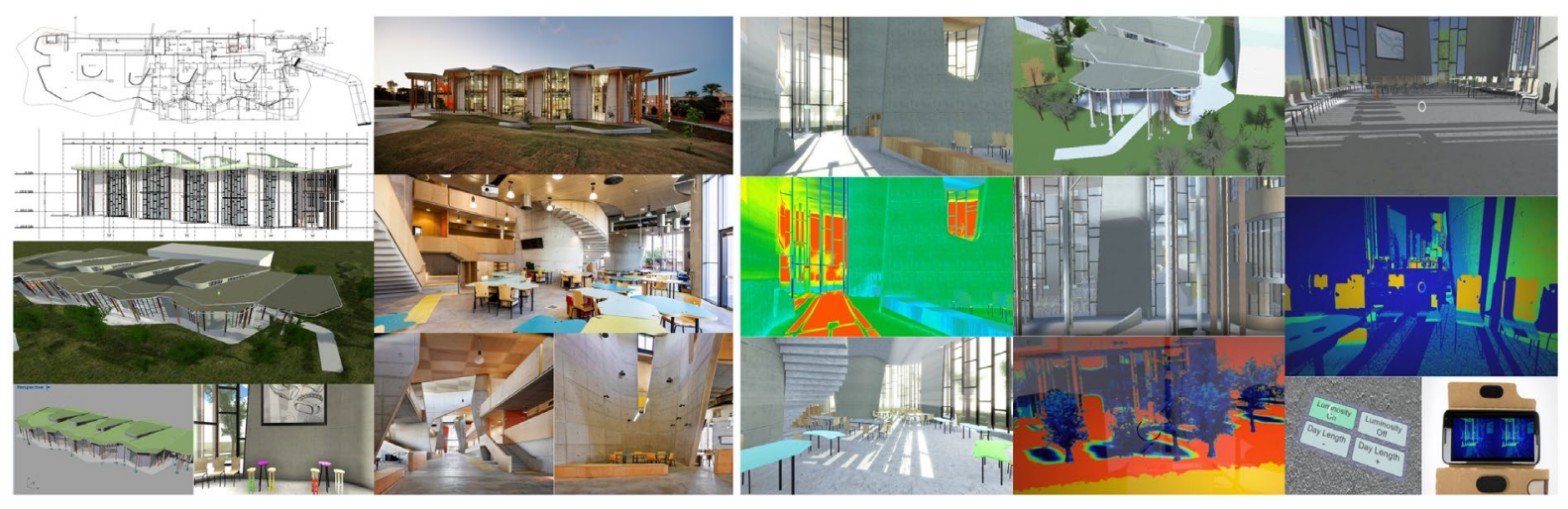

Figure 2. Comparative visualisations in the form of 2D orthographic views, physical buildings, virtual reality, augmented reality and mobile (Adapted from Birt and Cowling 2018) 
In particular, a key focus of MRMLA is the choice of how physical objects will be represented to provide characteristics such as manipulability, navigability, memorability, flexibility and other factors. To this end, marker technology is often used to recognise objects in physical space and augment them with a digital overlay. Marker technology uses the camera on a mixed reality device to recognise specific patterns and shapes that are unique to an object; these can then be replaced or augmented with digital items as illustrated by a learning example in Birt and Cowling ( 2017) (see Figure 3).

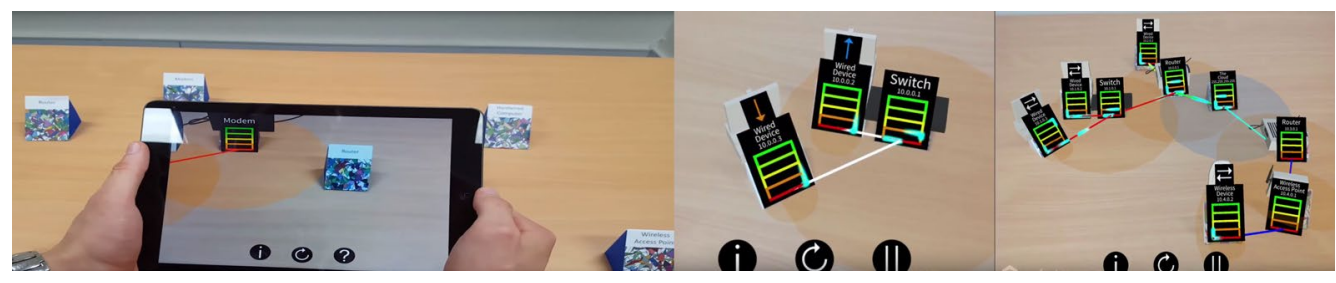

Figure 3. Example of mixed reality marker technology and digital overlay (Adapted from Birt and Cowling 2017)

Another approach to mapping digital objects onto a physical space is the use of depth sensing camera technology to generate a point cloud representing the physical world; this point cloud can then be used to place digital objects over and around physical items viewed through the camera system. In addition, Geolocation via Global Positioning System (GPS) is also often used to provide permanence for digital objects in a physical location. This can be supplemented with other forms of location-based services (via Bluetooth Low Energy (BLE) based systems) such as iBeacons and Eddystone technologies to assist with internal proximity and location.

When working with digital objects in a true mixed reality environment, there is intrinsic value in moving left and right across the virtuality continuum to provide users with a seamless experience. Often mixed reality simulations start with a purely physical view of the world that is used to orient the user, and in which the user then scans the environment (in the case of depth sensing) or places an object in front of the camera (in the case of marker based object recognition). The system then moves right across the continuum, providing an enhanced digital overlay based on the physical environment, or even turning the camera off to allow the user to focus on the digital objects (providing an Augmented Virtuality environment, as per the continuum). If the user moves location or loses track of the object, the system will move left across the continuum to allow for physical object viewing and reorientation. In this way, a more natural experience can be obtained for the user.

\section{Multimodal Mixed Reality Learning}

From a learner's perspective, MRMLA presents a unique opportunity to engage with content that would otherwise be hard for students to interact with. Examples include health applications such as visualization of anatomical structures, skill development applications such as practice in the use of tools and techniques, and visualization and simulation examples, such as being able to deconstruct a mechanical device or simulate a dangerous situation such as a bridge collapse.

Further, whilst some of these applications could be completed in a physical or virtual environment, MRMLA provides an opportunity for students to self-direct the simulation or application experience in an immersive, repeatable manner, where the learner directs (makes the choice) of how the learning plays out, what angle it is viewed from, and how the digital objects interact with the physical world. An example of this can be seen in figure 4 which shows the learner engaging in selfdirected mixed reality learning for training in paramedic airways management. (Birt, Moore and Cowling 2017). 


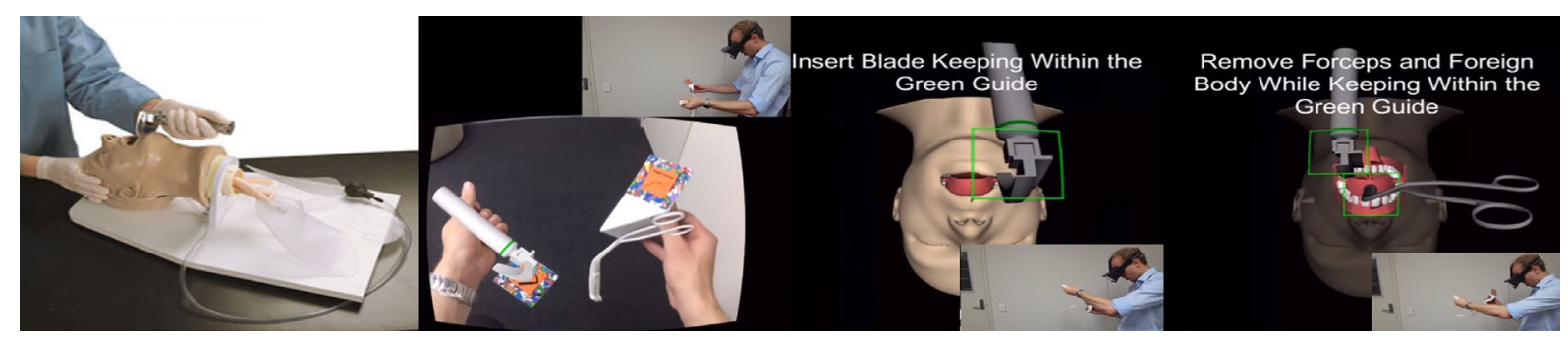

Figure 4. Examples of MRMLA from the self-directed perspective of the learner (Adapted from Birt, Moore and Cowling 2017)

An important consideration in this area is ensuring that data is presented in a multi coded (modal) way. This multimodal research is best captured in The Cambridge handbook of multimedia learning (Mayer, 2014), where multiple authors highlight the effects of multi coding, multimedia learning and cognitive theory and its positive effect on learning. In part, all visualizations must prime the learner's perception (asking "why do learners care?") draw on prior knowledge (affordance theory), avoid working memory overload (cognitive load theory) through specific learning objectives, provide multiple presentation modalities, move learners from shallow to deeper learning and allow learners the opportunity to apply and build their own mental models.

A true mixed reality system gives students the ability to experience a learning simulation using more than one modality including visual, auditory and haptic (touch) information to provide an immersive experience across multiple modes of learning. Given the nature of the interactions the learner is then primed and given correct learning design and affordances for the technology, providing the multiple modalities required for deeper learning enhancing the learners mental model. This in turn leads to engaged students and learning effectiveness.

\section{Spatial Learning Analytics}

Taking as given the value of a multimodal learning environment, MRMLA provides an opportunity to collect a new set of learning analytics through this multimodal process. Learning analytics is a field that concentrates on collecting relevant data from students during the learning process, analysing this data, and coming up with information that can be used to inform the student learning. Multimodal learning analytics seeks to investigate how combinations of various data types such as text, audio, camera, physiological and gestural data sourced through traditional and new emerging technology methods can be combined to more holistically understand both the learner and learning environment (Di Mitri, Schneider, Specht and Drachsler, 2018).

Mixed reality provides the opportunity to support and extend this learning analytics paradigm by providing ample opportunity for collecting additional analytics that we call spatial learning analytics. This includes the relative positioning of digital objects in a physical space, as well as how those objects are manipulated over the time given for the simulation. In situations where objects have interactive elements, a record can be taken of how these interactive elements are operated, as well as the order in which they are operated and whether operations are repeated. Further data about where the student is looking during the simulation, which objects are manipulated (in multi object scenarios) and how they interact with the overall simulation world can also be collected to provide a comprehensive picture of the student learning experience.

In certain situations, this data can then be extended to provide a new type of examination environment for students. By removing components and providing scaffolding instructions, students can be examined inside a mixed reality system to assess their understanding of the course content. Further, in addition to collecting data on student answers, spatial learning based examinations can 
also provide additional data from the learning analytics system to understand how students got to the answer that they provided.

For example, a student asked to identify the different components of a digital heart would be able to provide answers to questions, but then in addition these answers could be anchored to a set of learning analytics showing how the student arrived at this answer (via rotation of a digital heart model, selection of the wrong sections etc). In the style of checking a students working, this can then be used to allocate marks and more importantly enhance the learning process and offer new methods for creating more authentic assessment. An example of this can be seen in figure 5 which shows the learner engaging in MR and the dashboard system providing the learning stakeholders with MLA, enhancing observation and decision making (Birt, Clare and Cowling, 2019).

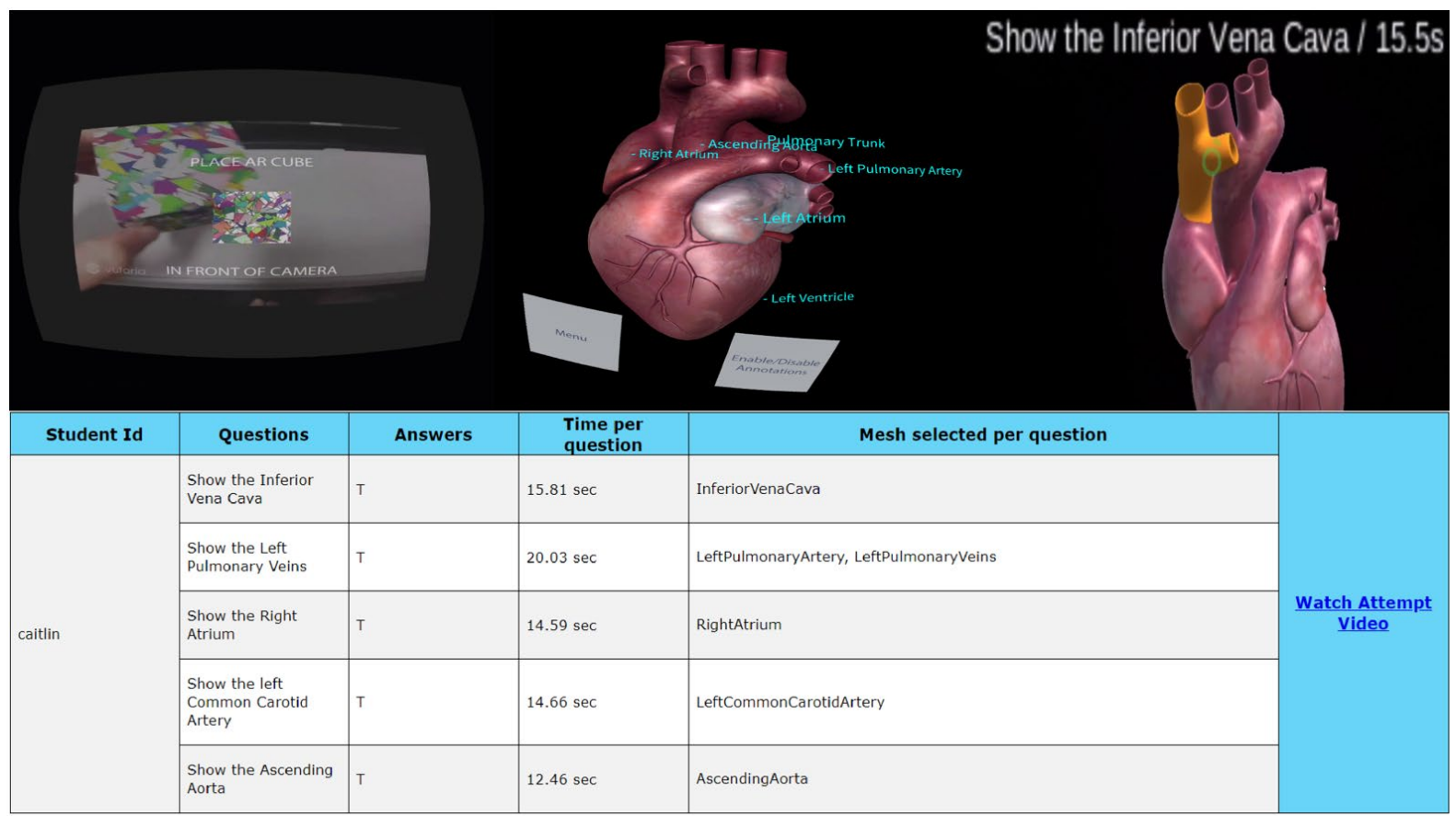

Figure 5. Collecting learning analytics from the observation and learner examination of a MR heart model (Adapted from Birt, Clare and Cowling 2019)

\section{Collaborative Mixed Reality \& Comparative Visualization}

Spatial Learning Analytics provide a mechanism for monitoring student performance, but another factor that cannot be overlooked is communication and feedback. A good spatial learning system should not only provide feedback via learning analytics after the simulation is concluded, but also during the simulation to change a students practice. One approach to do this is to allow students to collaborate in a shared MR space.

Sometimes referred to as Collaborative Augmented Reality or Blended Reality, this mechanism for a Multi-User Mixed Reality collaboration allows for seamless stakeholder collaboration. Building on work by Bower, Lee and Dalgarno (2017) and others on student collaboration in a virtual world, a Multiuser MR system allows for seamless communication between students either within the same physical space, or at a distance via networking technology.

Learning analytics data can then be used to provide augmentation that enhances the feedback loop provided by this communication, and in a true mixed reality system this communication should be device agnostic and work on any compatible mixed reality handset or headset. With this in mind, an 
important consideration for MRMLA is how these different systems provide different affordances for student feedback on a mixed reality simulation. Called comparative visualization, this process recognizes and exposes a student to different models of a visualization with different context and information to inform their learning process.

For example, a student viewing a virtual reality view of a building may be able to visit parts of that building that are not accessible in a physical space, whilst a student viewing an augmented reality visualization of the same building can see contextually relevant analytics data for different parts of the building (like maximum and minimum predicted temperatures for a component of the structure), and a student using true mixed reality might use a digital object to view a scaled digital model of the building that provides an overall holistic view and greater navigability and manipulability characteristics. In this way, spatial learning analytics and mixed reality can be used to provide both single and multi-user feedback on the learning system in turn working together to inform the learning stakeholders and overall learning environment.

\section{Mixed Reality Machine Learning}

Finally, an emerging area of interest in the mixed reality space is artificial intelligence (Al). Building on the possibility of a multimodal, multi-user, collaborative, comparative, learning analytics driven system, Al is the clear next step to understand how student feedback data can be used and fed back into the learning ecosystem.

Al can be used in a number of ways to address better student learning. Firstly, analytics data that is gathered in a MRMLA system can be analyzed in real-time and trends can be identified to provide real-time feedback to students about their learning. This builds on existing learning analytics research (Di Mitri, Schneider, Specht and Drachsler, 2018) with the addition of spatial learning data to understand the student learning process.

In the guise of a guide on the side, the Al system can watch a student and provide prompts and feedback to influence their learning. For instance, a student looking at a model of the heart in mixed reality to identify a particular component might be coached to turn the heart a certain way or to view from a certain angle to guide them towards the correct answer. Al can be used to determine what these instructions can and should be for the individual learner. Further, Al can also be used to analyze feedback and collaboration data after a session. Semantic and linguistic analysis using Al can be conducted to identify themes of interest and patterns in student usage that can then be summarized and aggregated as a form of summative feedback for future learning sessions.

For example, a student session around an architectural building might be recorded by a MRMLA system and then transcribed and analyzed to understand what they discussed, where they felt they had difficulty, and how the session progressed. This data can then be fed into a design research cycle to update the mixed reality intervention for the next learning session.

Throughout this process, it's important to keep in mind aspects of privacy and security of student data. To maintain traction with students, it's important that data only be used in an aggregate form, and that proper security controls are maintained. Learning should remain trusted, and students should not be afraid to ask stupid questions. Only with proper security and privacy controls can this be assured.

\section{Conclusion}

As an emerging field, mixed reality appears to have a lot of potential. Building on existing definitions of mixed reality, it now appears to be moving towards a new learning paradigm, where teachers recognize the value of multimodal representation, spatial learning analytics, collaboration \& 
comparative visualization, and the use of artificial intelligence for data analytics and learning analysis.

In terms of Al, these systems are algorithms and are based on data points and they can be biased on certain features. Big data algorithms generalise and classify the underpinning data meaning that if the data is biased the generalised data is also biased. As reliance on this data becomes more and more essentially, we could run the risk that the biased data, ignores individual learner differences; individuals become just a point on a numerical weighted graph. When you start to delve into the philosophical and ethical dilemmas of $\mathrm{Al}$ and big data things get very difficult especially from an education perspective where the individual must always be considered.

Though early days, it's clear that through a combination of these factors, a mixed reality multimodal learning analytics MRMLA system has the potential to transform education.

\section{References:}

Birt, J. R., \& Cowling, M. (2017). Toward future 'mixed reality' learning spaces for STEAM education. International Journal of Innovation in Science and Mathematics Education, 25(4), 1-16. https://openjournals.library.sydney.edu.au/index.php/CAL/article/download/12173/11405.

Birt, J. R., \& Cowling, M. A. (2018). Assessing mobile mixed reality affordances as a comparative visualization pedagogy for design communication. Research in Learning Technology, 26, 2128. https://doi.org/10.25304/rlt.v26.2128.

Birt, J., Moore, E., \& Cowling, M. (2017). Improving paramedic distance education through mobile mixed reality simulation. Australasian Journal of Educational Technology, 33(6), 69-83. https://doi.org/10.14742/ajet.3596.

Birt, J. R., Clare, D., \& Cowling, M. A. (2019). Piloting Multimodal Learning Analytics using Mobile Mixed Reality in Health Education. In D. Duque, J. White, N. Rodrigues, J. L. Vilaca, \& N. Dias (Eds.), 2019 IEEE 7th International Conference on Serious Games and Applications for Health (SeGAH) (pp. 1-6). [8882435] (2019 IEEE 7th International Conference on Serious Games and Applications for Health, SeGAH 2019). https://doi.org/10.1109/SeGAH.2019.8882435.

Bower, M., Lee, M. J., \& Dalgarno, B. (2017). Collaborative learning across physical and virtual worlds: Factors supporting and constraining learners in a blended reality environment. British Journal of Educational Technology, 48(2), 407-430. https://doi.org/10.1111/bjet.12435.

Di Mitri, D., Schneider, J., Specht, M., \& Drachsler, H. (2018). From signals to knowledge: A conceptual model for multimodal learning analytics. Journal of Computer Assisted Learning, 34(4), 338-349. https://doi.org/10.1111/jcal.12288.

Mayer, R. (Ed.). (2014). The Cambridge handbook of multimedia learning (Cambridge handbooks in psychology). Cambridge: Cambridge University Press. https://doi.org/10.1017/CBO9781139547369.

Milgram, P., \& Kishino, F. (1994). A taxonomy of mixed reality visual displays. IEICE Transactions on Information and Systems, 77(12), 1321-1329.

\section{Cross References}

1. Design-Based Research Methodology that Utilises Mixed Reality (MR) Technologies to Educate People About High-Risk (or Extreme) Situations

2. Human-Computer Interaction Visualisation in Mixed Reality

3. Mixed Reality and Embodied Cognition

4. Mixed Reality in Health Education, Potential of

5. Real Reality in Mixed Reality Innovation in Education 